\title{
"Schrottinfos" aus dem Web erschweren den Arzt-Patienten-Dialog
}

\author{
Geht es um die Diagnose oder Therapie der eigenen Erkrankung, schauen \\ immer mehr Patienten erst ins Internet, bevor ihr Weg in die Arztpraxis führt. \\ Doch das Wissen aus dem Web ist trügerisch, warnen Experten. Sie betonen: \\ Einen Dialog zwischen Ärzten und Patienten auf Augenhöhe werde es nie geben.
}

— Dialog auf Augenhöhe, mündige Patienten: Solche Schlagworte gehen seit Jahren durch die Medien, wenn es um das Verhältnis zwischen Patienten und Ärzten geht. Für Prof. Ingrid Mühlhauser von der Universität Hamburg sind und bleiben das Wunschvorstellungen. „Der Dialog zwischen Ärzten und Patienten wird nie auf Augenhöhe stattfinden. Das ist eine Illusion", sagte die Gesundheitswissenschaftlerin und Kongresspräsidentin der 13. Jahrestagung des Vereins Deutsches Netzwerk Evidenzbasierte Medizin (DNebM).

\section{Patienten müssen die Informationen gut sortieren}

Mühlhauser begründete ihre Einschätzung mit der Schwierigkeit, überhaupt an relevante Informationen zu gelangen, sie zu sortieren, zu gewichten und daraus die richtigen Schlüsse zu ziehen. „Patienten wollen zwar entscheiden. Dafür aber brauchen sie Informationen, die ihnen entweder fehlen, oder diese sind falsch oder irreführend“, so Mühlhauser. Das Internet spielt bei dieser Entwicklung eine zentrale Rolle.

„Wissen ohne Ende, aber keiner, der es sortiert", brachte die DNEbM-Vorsitzende Dr. Monika Lelgemann dieses Problem auf den Punkt. Gudrun Kemper von der deutschen Sektion der Breast Cancer Action spricht drastisch von „ungeprüften Schrottinfos, die eine Auseinandersetzung mit den Diagnose- und Therapiemöglichkeiten erschweren oder gar verhindern".
Bestehen Patientenverbände auf Unabhängigkeit, haben sie ein Finanzierungsproblem: Der Aufbau einer Infrastruktur kostet Geld. Das DNEbM bekräftigte, dass Patientenvertretungen finanziell so ausgestattet werden müssen, dass sie nicht auf Industriemittel angewiesen sind.

\section{Kaum Chancen für eine unabhängige Bewertung}

Dass Patientenverbände in die Lage versetzt werden sollten, medizinische Informationen unabhängig bewerten $\mathrm{zu}$ lassen, dafür ist nach Kempers Erfahrungen das Bewusstsein in Deutschland noch sehr schwach ausgeprägt. Kemper und andere Patientenvertreter sind aber sicher, dass die Chance auf einen Thera-

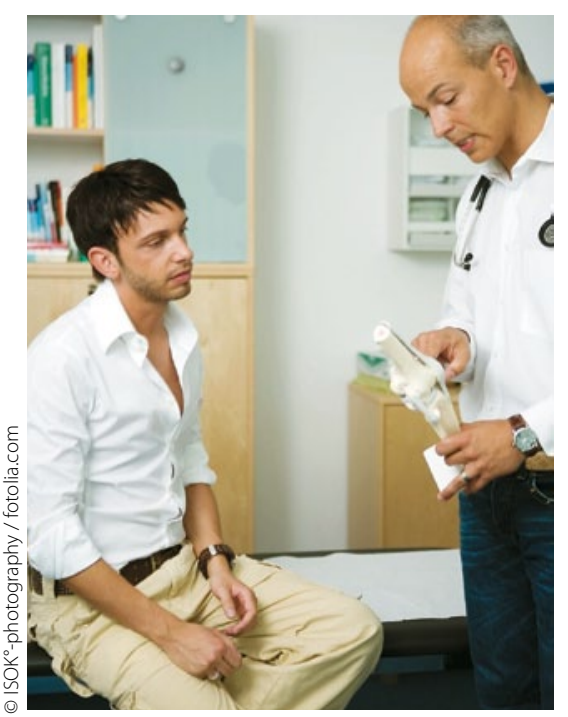

Zwischen Arzt und Patient wird es immer ein Informationsgefälle geben. pieerfolg steigt, wenn die Patienten einbezogen werden und an medizinischen Entscheidungen mitwirken können.

Neben der Industrie, die nach Ansicht vieler DNEbM-Vertreter unabhängige Entscheidungen erschwere, sehen Patientenvertreter auch die Ärzte in einer Schlüsselrolle. Diese Erfahrung hat z. B. Udo Ehrmann von der Selbsthilfe Prostata gemacht. Er hat in Kooperation mit dem Deutschen Krebsinformationszentrum und dem AOK-Bundesverband eine Website zum PSA-Test initiiert, die eine internetgestützte Entscheidungshilfe bietet (www.psa-entscheidungshilfe.de).

Diese soll die ärztliche Aufklärung zur Krebsvorsorge bei Männern unterstützen, stößt nach Erfahrungen Ehrmanns bei vielen Urologen aber auf Skepsis. Ehrmann ist überzeugt, dass sich viele Patienten selbstständig entscheiden und sich nicht ausschließlich auf den Rat des Arztes verlassen wollen.

\section{Viele Ärzte haben Vorbehalte}

Diese Haltung ist eines der wichtigsten Ziele des Netzwerks, das sich für eine partizipative Entscheidungsfindung einsetzt, damit Patienten sich entsprechend ihrer persönlichen Präferenzen für oder auch gegen medizinische Maßnahmen entscheiden können. Manche Ärzte haben damit nach Erfahrungen des Netzwerks aber ein Problem: Laut Mühlhauser wollen viele Ärzte nicht, dass Patienten jede Information kennen.

Doch selbst mit Vorliegen und Verfügbarkeit unabhängiger Informationen ist Mühlhauser sicher, dass die Augenhöhe zwischen Arzt und Patient schon wegen der unterschiedlichen Vorbildung nicht zu erreichen sein wird.

DIRK SCHNACK : 